\title{
Dynamic behaviors of soliton solutions for a three-coupled Lakshmanan-Porsezian-Daniel model
}

\author{
Bei-Bei $\mathrm{Hu}^{a, b, *}$, Ji Lin ${ }^{a, *}$, Ling Zhang ${ }^{b}$ \\ a.Department of Physics, Zhejiang Normal University, Jinhua, 321004, China \\ b.School of Mathematics and Finance, Chuzhou University, Anhui, 239000, China
}

September 9, 2021

\begin{abstract}
In this paper, we use the Riemann-Hilbert $(\mathrm{RH})$ approach to examine the integrable three-coupled Lakshmanan-Porsezian-Daniel (LPD) model, which describe the dynamics of alpha helical protein with the interspine coupling at the fourth-order dispersion term. Through the spectral analysis of Lax pair, we construct the higher order matrix $\mathrm{RH}$ problem for the three-coupled LPD model, when the jump matrix of this particular $\mathrm{RH}$ problem is a $4 \times 4$ unit matrix, the exact $\mathrm{N}$-soliton solutions of the three-coupled LPD model can be exhibited. As special examples, we also investigate the nonlinear dynamical behaviors of the single-soliton, two-soliton, three-soliton and breather soliton solutions. Finally, an integrable generalized N-component LPD model with its linear spectral problem is discussed.
\end{abstract}

PACS numbers:: 02.30.Ik, 02.20.Sv.

Mathematics Subject Classification (2010): 35C08, 35Q15, 37K10, 45D05.

Keywords: Lax pair; Riemann-Hilbert approach; three-coupled Lakshmanan-PorsezianDaniel model; soliton solutions; dynamic behaviors.

${ }^{*}$ Corresponding authors. E-mails: hubsquare@chzu.edu.cn(B.-B. Hu); linji@zjnu.edu.cn(J. Lin) 


\section{Introduction}

Since the nonlinear evolution equations can be widely used to describe some of the physics physical phenomena, such as nonlinear optical, quantum mechanics, fluid physics, plasma physics, etc. The research on the method of solving nonlinear evolution equations becomes a challenging and vital task, and it has attracted more and more people's attention. With the development of soliton theory, a series of methods for solving nonlinear development equations are proposed, such as the inverse scattering method [1], the Hirota's bilinear method [2], the Bäcklund transformation method [3], the Darboux transformation (DT) method [4] and others [5, 6, 7, 8]. Based on these available methods, we have obtained a series of solutions of nonlinear evolution equations, including compaton solution, peakon solution, periodic sharp wave solution, Lump solution, breather solution, bright soliton, dark soliton, rogue waves, etc. These solutions can further help to understand natural phenomena and laws. In recent years, more and more mathematical physicists have begun to pay attention to Riemann-Hilbert $(\mathrm{RH})$ approach $[9,10]$, which is a new powerful method for solving integrable linear and nonlinear evolution equations $[11,12,13,14,15,16,17$, $18,19,20,21,22,23,24,25,26,27]$. The main idea of this method is to establish a corresponding matrix RH problem on the Lax pair of integrable equations. Furthermore, the $\mathrm{RH}$ approach is also an effective way to examine the initial boundary value problems $[28,29,30,31,32]$ and the long-time asymptotic behavior [33, 34] of the integrable nonlinear evolution equations.

The Lakshmanan-Porsezian-Daniel (LPD) model [35] is one of the most paramount integrable systems in mathematics and physics which reads

$$
i q_{t}+q_{x x}+2 q|q|^{2}+\varepsilon\left(q_{x x x x}+8|q|^{2} q_{x x}+2 q^{2} q_{x x}^{*}+6 q^{*} q_{x}^{2}+4 q q_{x} q_{x}^{*}+6 q|q|^{4}\right)=0 .
$$

This model can not only simulate the nonlinear transmission and interaction of ultrashort pulses in high-speed optical fiber transmission systems, but also describe the nonlinear spin excitation phenomenon of a one-dimensional Heisenberg ferromagnetic chain with octopole and dipole interactions [36]. When $\varepsilon=0$, Eq.(1.1) can be reduced to the famous nonlinear schrödinger (NLS) equation. However, a slice of phenomena have been observed by experiment which cannot be explained by Eq.(1.1). For example, the dynamics of the alpha helical protein with the interspine coupling at the fourth-order dispersion term. In 
order to explain these phenomena, the three-component case was introduced as follows $[37,38,39]$

$$
\begin{aligned}
i q_{\alpha, t} & +q_{\alpha, x x}+2 \sum_{k=1}^{3}\left|q_{k}\right|^{2} q_{\alpha}+\varepsilon\left[q_{\alpha, x x x x}+2 \sum_{k=1}^{3}\left|q_{k, x}\right|^{2} q_{\alpha}\right. \\
& +2 \sum_{k=1}^{3} q_{k} q_{k, x}^{*} q_{\alpha, x}+6 \sum_{k=1}^{3} q_{k}^{*} q_{k, x} q_{\alpha, x}+4 \sum_{k=1}^{3}\left|q_{k}\right|^{2} q_{\alpha, x x} \\
& \left.+4 \sum_{k=1}^{3} q_{k}^{*} q_{k, x x} q_{\alpha}+2 \sum_{k=1}^{3} q_{k} q_{k, x x}^{*} q_{\alpha}+6\left(\sum_{k=1}^{3}\left|q_{k}\right|^{2}\right)^{2} q_{\alpha}\right]=0
\end{aligned}
$$

where $q_{\alpha}(x, t),(\alpha=1,2,3)$ represent amplitude of molecular excitation in the $\alpha$-th spine, the subscripts $x$ and $t$ represent the partial derivatives with respect to the scaled space variable and retarded time variable, respectively, the $*$ denotes the complex conjugate, and $\varepsilon$ is a real parameter standing for the strength of higher-order linear and nonlinear effects. When $\varepsilon=0$, Eq.(1.2) can be reduced to the three-component NLS equation [40, 41]. Indeed, the three-coupled LPD model (1.2) is a completely integrable model, and quiet a few properties have been investigated, for instance, the Lax pair [37], the Hirota bilinear forms, the soliton solutions by the DT method and the binary Bell-polynomial approach [38], the semirational rogue waves by the generalized DT method [39]. In this paper, we aim to investigate the soliton solutions of three-coupled LPD model (1.2) via the RH approach, and discuss the dynamic behavior of the soliton solutions.

The organization of this paper is as follows. In section 2, we will construct a specific $\mathrm{RH}$ problem based on the inverse scattering transformation. In section 3 , we compute Nsoliton solutions of the three-coupled LPD model (1.2) from a specific RH problem, which possesses the identity jump matrix on the real axis. In section 4, the spatial structures and collision dynamics behaviors of soliton solutions are examined. In section 5, as a promotion, we briefly explain an integrable generalized multi-component LPD model .

\section{The Riemann-Hilbert problem}

The three-coupled LPD model (1.2) possesses the following Lax pair [39]

$$
\Phi_{x}=U(x, t, \xi) \Phi=\left(i \xi \sigma_{4}+M(x, t)\right) \Phi
$$




$$
\Phi_{t}=V(x, t, \xi) \Phi=\left(-8 i \varepsilon \xi^{4} \sigma_{4}+2 i \xi^{2} \sigma_{4}+Q(x, t, \xi)\right) \Phi
$$

where $\xi$ is a complex spectral parameter, $\Phi=\left(\Phi_{1}, \Phi_{2}, \Phi_{3}, \Phi_{4}\right)^{T}$ is a vector function, the $4 \times 4$ matrices $\sigma_{4}, M(x, t)$ are defined by

$$
\sigma_{4}=\left(\begin{array}{cccc}
1 & 0 & 0 & 0 \\
0 & -1 & 0 & 0 \\
0 & 0 & -1 & 0 \\
0 & 0 & 0 & -1
\end{array}\right), M(x, t)=\left(\begin{array}{cccc}
0 & q_{1}^{*} & q_{2}^{*} & q_{3}^{*} \\
q_{1} & 0 & 0 & 0 \\
q_{2} & 0 & 0 & 0 \\
q_{3} & 0 & 0 & 0
\end{array}\right)
$$

and $Q(x, t, \xi)=Q_{4 \times 4}$ is a matrix-value function defined by

$$
\begin{aligned}
Q(x, t, \xi)= & -8 i \varepsilon M \xi^{3}+4 \varepsilon\left(i M^{2} \sigma_{4}-\sigma_{4} M_{x}\right) \xi^{2}+2\left(i \varepsilon M_{x x}+2 i \varepsilon M^{3}\right. \\
& \left.-\varepsilon\left(M M_{x}-M_{x} M\right)+i M\right) \xi+\varepsilon \sigma_{4} M_{x x x}-i \varepsilon \sigma_{4}\left(M M_{x x}+M_{x x} M\right) \\
& -3 i \varepsilon \sigma_{4} M^{4}-i \sigma_{4} M^{2}+i \varepsilon M_{x}^{2} \sigma_{4}+3 \varepsilon \sigma_{4}\left(M^{2} M_{x}+M_{x} M^{2}\right)+\sigma_{4} M_{x}
\end{aligned}
$$

Eq.(2.1a)-(2.1b) is rewritten as

$$
\begin{aligned}
& \Phi_{x}-i \xi \sigma_{4} \Phi=M(x, t) \Phi \\
& \Phi_{t}+\left(8 i \varepsilon \xi^{4} \sigma_{4}-2 i \xi^{2} \sigma_{4}\right) \Phi=Q(x, t, \xi) \Phi
\end{aligned}
$$

$\tilde{A}(x, t, \xi)=e^{\left(i \xi x-8 i \varepsilon \xi^{4} t+2 i \xi^{2} t\right) \sigma_{4}}$ is a solution for Eq.(2.4a)-(2.4b), by setting $\Phi(x, t, \xi)=$ $\Psi(x, t, \xi) \tilde{A}(x, t, \xi)$, we have the following Lax pair forms

$$
\begin{aligned}
& \Psi_{x}-i \xi\left[\sigma_{4}, \Psi\right]=M(x, t) \Psi \\
& \Psi_{t}+\left(8 i \varepsilon \xi^{4}-2 i \xi^{2}\right)\left[\sigma_{4}, \Psi\right]=Q(x, t, \xi) \Psi
\end{aligned}
$$

Now, we construct two Jost solutions $\Psi_{ \pm}=\Psi_{ \pm}(x, \xi)$ of Eq.(2.5a)

$$
\begin{aligned}
& \Psi_{+}=\left(\left[\Psi_{+}\right]_{1},\left[\Psi_{+}\right]_{2},\left[\Psi_{+}\right]_{3},\left[\Psi_{+}\right]_{4}\right), \\
& \Psi_{-}=\left(\left[\Psi_{-}\right]_{1},\left[\Psi_{-}\right]_{2},\left[\Psi_{-}\right]_{3},\left[\Psi_{-}\right]_{4}\right),
\end{aligned}
$$

with the boundary conditions

$$
\Psi_{+} \rightarrow \mathrm{I}, x \rightarrow+\infty
$$




$$
\Psi_{-} \rightarrow \mathrm{I}, x \rightarrow-\infty
$$

where $\mathrm{I}=\operatorname{diag}\{1,1,1,1\}$ is a $4 \times 4$ identity matrix, $\left[\Psi_{ \pm}\right]_{n}(n=1,2,3,4)$ denote the $n$ th column vector of $\Psi_{ \pm}$. Indeed, for $\xi \in \mathbb{R}$, the two Jost solutions $\Psi_{ \pm}=\Psi_{ \pm}(x, \xi)$ are determined by Volterra integral equations as follows

$$
\begin{aligned}
& \Psi_{+}(x, \xi)=\mathrm{I}-\int_{x}^{+\infty} e^{i \xi \hat{\sigma}_{4}(x-\zeta)} M(\zeta) \Psi_{+}(\zeta, \xi) d \zeta \\
& \Psi_{-}(x, \xi)=\mathrm{I}+\int_{-\infty}^{x} e^{i \xi \hat{\sigma}_{4}(x-\zeta)} M(\zeta) \Psi_{-}(\zeta, \xi) d \zeta
\end{aligned}
$$

where $\hat{\sigma}_{4}$ represents a matrix operator (see [28]).

If $\mathbb{C}_{+}$and $\mathbb{C}_{-}$denote the upper half $\xi$-plane and the lower half $\xi$-plane, respectively, then $\left[\Psi_{-}\right]_{1},\left[\Psi_{+}\right]_{2},\left[\Psi_{+}\right]_{3}$ and $\left[\Psi_{+}\right]_{4}$ admit analytic extensions to $\mathbb{C}_{-},\left[\Psi_{+}\right]_{1},\left[\Psi_{-}\right]_{2},\left[\Psi_{-}\right]_{3}$ and $\left[\Psi_{-}\right]_{4}$ admit analytic extensions to the $\mathbb{C}_{+}$. Furthermore, the determinants of $\Psi_{ \pm}$are constants for all $x$ because of the Abel's identity and $\operatorname{Tr}(M)=0$. It follows from the boundary conditions Eq.(2.8) that

$$
\operatorname{det} \Psi_{ \pm}=1, \quad \xi \in \mathbb{R}
$$

Next, we introduce a new function $A(x, \xi)=e^{i \xi \sigma_{4} x}$, then, Eq.(2.5a) exists two fundamental matrix solutions $\Psi_{+} A$ and $\Psi_{-} A$ which satisfy the following relationship

$$
\Psi_{-} A=\Psi_{+} A \cdot S(\xi), \quad \xi \in \mathbb{R}
$$

where $S(\xi)$ is a $4 \times 4$ scattering matrix. From Eqs.(2.9)-(2.10), we know

$$
\operatorname{det} S(\xi)=1
$$

Let $x \rightarrow+\infty$, we have the $4 \times 4$ scattering matrix $S(\xi)$

$$
S(\xi)=\left(s_{i j}\right)_{4 \times 4}=\lim _{x \rightarrow+\infty} A^{-1} \Psi_{+}^{-1} \Psi_{-} A=\mathrm{I}+\int_{-\infty}^{+\infty} e^{-i \xi \hat{\sigma}_{4} \zeta} M \Psi_{-} d \zeta, \xi \in \mathbb{R} .
$$

It follows from the analytic property of $\Psi_{-}$that $s_{11}$ allows analytic extensions to $\mathbb{C}_{-}$, the others $s_{i j}, i, j \neq 1$ allow analytic extensions to $\mathbb{C}_{+}$. 
In order to discuss behavior of Jost solutions for large $\xi$, we assume that

$$
\Psi=\Psi_{0}+\frac{\Psi_{1}}{\xi}+\frac{\Psi_{2}}{\xi^{2}}+\frac{\Psi_{3}}{\xi^{3}}+\frac{\Psi_{4}}{\xi^{4}}+\cdots, \quad \xi \rightarrow \infty,
$$

substituting the above expansion Eq.(2.13) into the Eq.(2.5a) and comparing the coefficients of the same order of $\xi$ yields

$$
\begin{aligned}
& O\left(\xi^{1}\right):-i\left[\sigma_{4}, \Psi_{0}\right]=0, \\
& O\left(\xi^{0}\right): \Psi_{0, x}-i\left[\sigma_{4}, \Psi_{1}\right]-M \Psi_{0}=0, \\
& O\left(\xi^{-1}\right): \Psi_{1, x}-i\left[\sigma_{4}, \Psi_{2}\right]-M \Psi_{1}=0,
\end{aligned}
$$

from $O\left(\xi^{1}\right)$ and $O\left(\xi^{0}\right)$, we have

$$
i\left[\sigma_{4}, \Psi_{1}\right]=-M \Psi_{0}, \Psi_{0, x}=0 .
$$

On the one hand, we define another new Jost solution for Eq.(2.5a) by

$$
H_{+}=\left(\left[\Psi_{-}\right]_{1},\left[\Psi_{+}\right]_{2},\left[\Psi_{+}\right]_{3},\left[\Psi_{+}\right]_{4}\right),
$$

taking Eq.(2.6a)-(2.6b) into Eq.(2.10) gives rise to

$$
\begin{aligned}
& \left(\left[\Psi_{-}\right]_{1},\left[\Psi_{-}\right]_{2},\left[\Psi_{-}\right]_{3},\left[\Psi_{-}\right]_{4}\right)=\Psi_{+} A S(\xi) A^{-1} \\
& =\left(\left[\Psi_{+}\right]_{1},\left[\Psi_{+}\right]_{2},\left[\Psi_{+}\right]_{3},\left[\Psi_{+}\right]_{4}\right)\left(\begin{array}{cccc}
s_{11} & s_{12} e^{2 i \xi x} & s_{13} e^{2 i \xi x} & s_{14} e^{2 i \xi x} \\
s_{21} e^{-2 i \xi x} & s_{22} & s_{23} & s_{24} \\
s_{21} e^{-2 i \xi x} & s_{32} & s_{33} & s_{34} \\
s_{21} e^{-2 i \xi x} & s_{42} & s_{43} & s_{44}
\end{array}\right),
\end{aligned}
$$

from Eq.(2.17), we have

$$
\left[\Psi_{-}\right]_{1}=s_{11}\left[\Psi_{+}\right]_{1}+s_{21} e^{-2 i \xi x}\left[\Psi_{+}\right]_{2}+s_{31} e^{-2 i \xi x}\left[\Psi_{+}\right]_{3}+s_{41} e^{-2 i \xi x}\left[\Psi_{+}\right]_{4},
$$

then, $H_{+}$can be rewritten as the following matrix form

$$
\begin{aligned}
H_{+} & =\left(\left[\Psi_{-}\right]_{1},\left[\Psi_{+}\right]_{2},\left[\Psi_{+}\right]_{3},\left[\Psi_{+}\right]_{4}\right)=\Psi_{+} S_{+} \\
& =\left(\left[\Psi_{+}\right]_{1},\left[\Psi_{+}\right]_{2},\left[\Psi_{+}\right]_{3},\left[\Psi_{+}\right]_{4}\right)\left(\begin{array}{cccc}
s_{11} & 0 & 0 & 0 \\
s_{21} e^{-2 i \xi x} & 1 & 0 & 0 \\
s_{31} e^{-2 i \xi x} & 0 & 1 & 0 \\
s_{41} e^{-2 i \xi x} & 0 & 0 & 1
\end{array}\right),
\end{aligned}
$$


which is analytic for $\xi \in \mathbb{C}_{-}$and admits asymptotic behavior for very large $\xi$ as

$$
H_{+} \rightarrow \mathrm{I}, \xi \rightarrow+\infty, \xi \in \mathbb{C}_{-} .
$$

On the other hand, in order to obtain the analytic counterpart of $H_{+}$denoted by $H_{-}$ in $\mathbb{C}_{+}$, we consider the adjoint scattering equation of Eq.(2.5a) as follows

$$
G_{x}-i \xi\left[\sigma_{4}, G\right]=-G M,
$$

obviously, the inverse matrices $\Psi_{ \pm}^{-1}$ is defined as

$$
\left[\Psi_{+}\right]^{-1}=\left(\begin{array}{c}
{\left[\Psi_{+}^{-1}\right]^{1}} \\
{\left[\Psi_{+}^{-1}\right]^{2}} \\
{\left[\Psi_{+}^{-1}\right]^{3}} \\
{\left[\Psi_{+}^{-1}\right]^{4}}
\end{array}\right), \quad\left[\Psi_{-}\right]^{-1}=\left(\begin{array}{c}
{\left[\Psi_{-}^{-1}\right]^{1}} \\
{\left[\Psi_{-}^{-1}\right]^{2}} \\
{\left[\Psi_{-}^{-1}\right]^{3}} \\
{\left[\Psi_{-}^{-1}\right]^{4}}
\end{array}\right),
$$

which satisfy the adjoint equation $(2.20)$, where $\left[\Psi_{ \pm}^{-1}\right]^{n}(n=1,2,3,4)$ denote the $n$-th row vector of $\Psi_{ \pm}^{-1}$, then, $\left[\Psi_{+}^{-1}\right]^{1},\left[\Psi_{-}^{-1}\right]^{2},\left[\Psi_{-}^{-1}\right]^{3}$ and $\left[\Psi_{-}^{-1}\right]^{4}$ admit analytic extensions to $\mathbb{C}_{-}$, $\left[\Psi_{-}^{-1}\right]^{1},\left[\Psi_{+}^{-1}\right]^{2},\left[\Psi_{+}^{-1}\right]^{3}$ and $\left[\Psi_{+}^{-1}\right]^{4}$ admit analytic extensions to the $\mathbb{C}_{+}$.

In addition, it is not difficult to find that the inverse matrices $\Psi_{+}^{-1}$ and $\Psi_{-}^{-1}$ satisfy the following boundary conditions

$$
\begin{aligned}
& \Psi_{+}^{-1} \rightarrow \mathrm{I}, x \rightarrow+\infty \\
& \Psi_{-}^{-1} \rightarrow \mathrm{I}, x \rightarrow-\infty
\end{aligned}
$$

therefore, we can define

$$
H_{-}=\left(\begin{array}{c}
{\left[\Psi_{-}^{-1}\right]^{1}} \\
{\left[\Psi_{+}^{-1}\right]^{2}} \\
{\left[\Psi_{+}^{-1}\right]^{3}} \\
{\left[\Psi_{+}^{-1}\right]^{4}}
\end{array}\right)
$$

by similar techniques analysis, we can get that $H_{-}$is analytic in $\mathbb{C}_{+}$

$$
H_{-} \rightarrow \mathrm{I}, \xi \rightarrow-\infty, \xi \in \mathbb{C}_{+} .
$$

Assume that $R(\xi)=S^{-1}(\xi)$, we obtain

$$
\Psi_{-}^{-1}=A R(\xi) A^{-1} \Psi_{+}^{-1},
$$


taking Eq.(2.21)-(2.6b) into Eq.(2.25) leads to

$$
\left(\begin{array}{c}
{\left[\Psi_{-}^{-1}\right]^{1}} \\
{\left[\Psi_{-}^{-1}\right]^{2}} \\
{\left[\Psi_{-}^{-1}\right]^{3}} \\
{\left[\Psi_{-}^{-1}\right]^{4}}
\end{array}\right)=\left(\begin{array}{cccc}
r_{11} & r_{12} e^{2 i \xi x} & r_{13} e^{2 i \xi x} & r_{14} e^{2 i \xi x} \\
r_{21} e^{-2 i \xi x} & r_{22} & r_{23} & r_{24} \\
r_{31} e^{-2 i \xi x} & r_{32} & r_{33} & r_{34} \\
r_{41} e^{-2 i \xi x} & r_{42} & r_{43} & r_{44}
\end{array}\right)\left(\begin{array}{c}
{\left[\Psi_{+}^{-1}\right]^{1}} \\
{\left[\Psi_{+}^{-1}\right]^{2}} \\
{\left[\Psi_{+}^{-1}\right]^{3}} \\
{\left[\Psi_{+}^{-1}\right]^{4}}
\end{array}\right)
$$

from Eq.(2.26), we have

$$
\left[\Psi_{-}^{-1}\right]^{1}=r_{11}\left[\Psi_{+}^{-1}\right]^{1}+r_{12} e^{2 i \xi x}\left[\Psi_{+}^{-1}\right]^{2}+r_{13} e^{2 i \xi x}\left[\Psi_{+}^{-1}\right]^{3}+r_{14} e^{2 i \xi x}\left[\Psi_{+}^{-1}\right]^{4}
$$

then, $H_{+}$can be rewritten as the following matrix form

$$
H_{-}=\left(\begin{array}{l}
{\left[\Psi_{-}^{-1}\right]^{1}} \\
{\left[\Psi_{+}^{-1}\right]^{2}} \\
{\left[\Psi_{+}^{-1}\right]^{3}} \\
{\left[\Psi_{+}^{-1}\right]^{4}}
\end{array}\right)=R_{+} \Psi_{+}^{-1}=\left(\begin{array}{cccc}
r_{11} & r_{12} e^{2 i \xi x} & r_{13} e^{2 i \xi x} & r_{14} e^{2 i \xi x} \\
0 & 1 & 0 & 0 \\
0 & 0 & 1 & 0 \\
0 & 0 & 0 & 1
\end{array}\right)\left(\begin{array}{l}
{\left[\Psi_{+}^{-1}\right]^{1}} \\
{\left[\Psi_{+}^{-1}\right]^{2}} \\
{\left[\Psi_{+}^{-1}\right]^{3}} \\
{\left[\Psi_{+}^{-1}\right]^{4}}
\end{array}\right)
$$

In fact, these two matrix functions $H_{+}(x, \xi)$ and $H_{-}(x, \xi)$ can be used to construct the following $\mathrm{RH}$ problem:

- $H_{+}(x, \xi)$ and $H_{-}(x, \xi)$ are analytic for $\xi$ in $\mathbb{C}_{-}$and $\mathbb{C}_{+}$, respectively.

- $H_{+}(x, \xi)$ and $H_{-}(x, \xi)$ satisfy the following jumping relationship on the real $x$-axis

$$
H_{-}(x, \xi) H_{+}(x, \xi)=T(x, \xi), \quad \xi \in \mathbb{R}
$$

where

$$
T(x, \xi)=\left(\begin{array}{cccc}
1 & r_{12} e^{2 i \xi x} & r_{13} e^{2 i \xi x} & r_{14} e^{2 i \xi x} \\
s_{21} e^{-2 i \xi x} & 1 & 0 & 0 \\
s_{31} e^{-2 i \xi x} & 0 & 1 & 0 \\
s_{41} e^{-2 i \xi x} & 0 & 0 & 1
\end{array}\right)
$$

and $r_{11} s_{11}+r_{12} s_{21}+r_{13} s_{31}+r_{14} s_{41}=1$.

- $H_{ \pm} \rightarrow \mathrm{I}, \quad \xi \rightarrow \infty$. 
Furthermore, since $\Psi_{-}$satisfies the temporal part of spectral equation

$$
\Psi_{-, t}+\left(8 i \varepsilon \xi^{4}-2 i \xi^{2}\right)\left[\sigma_{4}, \Psi_{-}\right]=Q(x, t, \xi) \Psi_{-},
$$

from Eq.(2.10), we have

$$
\left(\Psi_{+} A S\right)_{t}+\left(8 i \varepsilon \xi^{4}-2 i \xi^{2}\right)\left[\sigma_{4}, \Psi_{+} A S\right]=Q(x, t, \xi) \Psi_{+} A S,
$$

for $x \rightarrow \infty$, assume that $q_{\alpha},(\alpha=1,2,3)$ are sufficient smoothness and fast decay, the matrix $Q(x, t, \xi) \rightarrow 0$ as $x \rightarrow \pm \infty$. Let $x \rightarrow+\infty$ in Eq.(2.31), we obtain

$$
S_{t}=-\left(8 i \varepsilon \xi^{4}-2 i \xi^{2}\right)\left[\sigma_{4}, S\right],
$$

which means that the scattering data $s_{11}, s_{22}, s_{33}, s_{23}, s_{32}$ are time independent

$$
s_{11, t}=s_{22, t}=s_{33, t}=s_{44, t}=s_{23, t}=s_{24, t}=s_{32, t}=s_{34, t}=s_{42, t}=s_{43, t}=0,
$$

and the other scattering data satisfy

$$
\begin{aligned}
& r_{12}(t, \xi)=r_{12}(0, \xi) e^{-4 i\left(4 \varepsilon \xi^{4}-\xi^{2}\right) t}, r_{13}(t, \xi)=r_{13}(0, \xi) e^{-4 i\left(4 \varepsilon \xi^{4}-\xi^{2}\right) t} \\
& r_{14}(t, \xi)=r_{14}(0, \xi) e^{-4 i\left(4 \varepsilon \xi^{4}-\xi^{2}\right) t}, s_{21}(t, \xi)=s_{21}(0, \xi) e^{4 i\left(4 \varepsilon \xi^{4}-\xi^{2}\right) t} \\
& s_{31}(t, \xi)=s_{31}(0, \xi) e^{4 i\left(4 \varepsilon \xi^{4}-\xi^{2}\right) t}, s_{41}(t, \xi)=s_{41}(0, \xi) e^{4 i\left(4 \varepsilon \xi^{4}-\xi^{2}\right) t}
\end{aligned}
$$

\section{The soliton solutions for three-coupled LPD model}

In this section, based on the $\mathrm{RH}$ problem constructed in Sec. 2, we would like to formulate the N-soliton solutions of three-coupled LPD model (1.2). In fact, the solution to this RH problem will not be unique unless the zeros of $\operatorname{det} H_{+}$and $\operatorname{det} H_{-}$in the upper and lower half of the $\xi$-plane are also specified, and the kernel structures of $H_{ \pm}$at these zeros are provided. It follows from the definitions of $H_{+}$and $H_{-}$that

$$
\operatorname{det} H_{+}(x, \xi)=s_{11}(\xi), \quad \operatorname{det} H_{-}(x, \xi)=r_{11}(\xi),
$$

which means that the zeros of $\operatorname{det} H_{+}$and $\operatorname{det} H_{-}$are the same as $s_{11}(\xi)$ and $r_{11}(\xi)$, respectively. Furthermore, the scattering data $s_{11}$ and $r_{11}$ are time independent (2.33), we can get that the roots of $s_{11}=0$ and $r_{11}=0$ are also time independent, since

$$
\sigma_{4} M(x, t) \sigma_{4}=-M(x, t), \quad M^{\dagger}(x, t)=M(x, t),
$$


where $\dagger$ represents the Hermitian of a matrix. It is easy to see that

$$
\Psi_{ \pm}(x, t,-\xi)=\sigma_{4} \Psi_{ \pm}(x, t, \xi) \sigma_{4}, \quad \Psi_{ \pm}^{-1}(x, t, \xi)=\Psi_{ \pm}^{\dagger}\left(x, t, \xi^{*}\right)
$$

according to reduction conditions given by Eqs.(2.6a)-(2.6b), we obtain

$$
S(-\xi)=\sigma_{4} S(\xi) \sigma_{4}, \quad R(\xi)=S^{-1}(\xi)=S^{\dagger}\left(\xi^{*}\right), \quad H_{-}(x, \xi)=H_{+}^{\dagger}\left(x, \xi^{*}\right) .
$$

Assume that $s_{11}$ enjoys $N \geq 0$ possible zeros denoted as $\left\{\xi_{m}, 1 \leq m \leq N\right\}$ in $\mathbb{C}_{-}, r_{11}$ enjoys $N \geq 0$ possible zeros denoted as $\left\{\widetilde{\xi}_{m}, 1 \leq m \leq N\right\}$ in $\mathbb{C}_{+}$. For the convenience of later discussion, without loss of generality, we suppose that all zeros $\left\{\left(\xi_{m}, \widetilde{\xi}_{m}\right), m=1,2, \ldots, N\right\}$ of $s_{11}$ and $r_{11}$ are simple zeros. In this case, each of $\operatorname{ker} H_{+}\left(\xi_{m}\right)$ and $\operatorname{ker} H_{-}\left(\widetilde{\xi}_{m}\right)$ includes only a single column vector $v_{m}$ and row vector $\widetilde{v}_{m}$, respectively, i.e.

$$
H_{+}\left(\xi_{m}\right) v_{m}=0, \quad \widetilde{v}_{m} H_{-}\left(\widetilde{\xi}_{m}\right)=0,
$$

because $H_{+}(\xi)$ is the solution of Eq.(2.5a), for large $\xi$, we suppose that $H_{+}(\xi)$ possesses the following asymptotic expansion

$$
H_{+}=\mathrm{I}+\frac{H_{+}^{(1)}}{\xi}+O\left(\xi^{-2}\right), \quad \xi \rightarrow \infty,
$$

taking Eq.(3.5) into Eq.(2.5a) and comparing $O(1)$ terms gets

$$
M=-i\left[\sigma_{4}, H_{+}^{(1)}\right]=\left(\begin{array}{cccc}
0 & -2 i\left(H_{+}^{(1)}\right)_{12} & -2 i\left(H_{+}^{(1)}\right)_{13} & -2 i\left(H_{+}^{(1)}\right)_{14} \\
2 i\left(H_{+}^{(1)}\right)_{21} & 0 & 0 & 0 \\
2 i\left(H_{+}^{(1)}\right)_{31} & 0 & 0 & 0 \\
2 i\left(H_{+}^{(1)}\right)_{41} & 0 & 0 & 0
\end{array}\right)
$$

then, the potential functions solutions $q_{1}(x, t), q_{2}(x, t)$ and $q_{3}(x, t)$ of the three-coupled LPD model (1.2) can be reconstructed by

$$
q_{1}(x, t)=2 i\left(H_{+}^{(1)}\right)_{21}, q_{2}(x, t)=2 i\left(H_{+}^{(1)}\right)_{31}, q_{3}(x, t)=2 i\left(H_{+}^{(1)}\right)_{41},
$$

where $H_{+}^{(1)}=\left(H_{+}^{(1)}\right)_{4 \times 4}$ and $\left(H_{+}^{(1)}\right)_{i j}$ are the $(i, j)$-entry of $H_{+}^{(1)}, i, j=1,2,3,4$.

In order to get the spatial evolutions for vectors $v_{m}(x, t)$, on the one hand, taking the derivative of equation $H_{+} v_{m}=0$ with respect to $x$ and with Eq.(2.5a), we obtain

$$
H_{+} v_{m, x}+i \xi_{m} H_{+} \sigma_{4} v_{m}=0
$$


thus

$$
v_{m, x}=-i \xi_{m} \sigma_{4} v_{m},
$$

on the other hand, taking the derivative of equation $H_{+} v_{m}=0$ with respect to $t$ and with Eq.(2.5b), we get

$$
H_{+} v_{m, t}-\left(8 i \varepsilon \xi_{m}^{4}-2 i \xi_{m}^{2}\right) H_{+} \sigma_{4} v_{m}=0,
$$

thus

$$
v_{m, t}=\left(8 i \varepsilon \xi_{m}^{4}-2 i \xi_{m}^{2}\right) \sigma_{4} v_{m},
$$

according to Eq.(3.9) and Eq.(3.11), we have

$$
v_{m}(x, t)=e^{-i \xi_{m} \sigma_{4} x+\left(8 i \varepsilon \xi_{m}^{4}-2 i \xi_{m}^{2}\right) \sigma_{4} t} v_{m 0} e^{\int_{x_{0}}^{x} \rho_{m}(y) d y+\int_{t_{0}}^{t} \omega_{m}(\tau) d \tau},
$$

where $v_{m 0}$ is constant, $\rho_{m}(y)$ and $\omega_{m}(\tau)$ are two scalar functions, at the same time, we have

$$
\widetilde{v}_{m}(x, t)=v_{m}^{\dagger}(x, t) .
$$

In order to obtain multi-soliton solutions for the three-coupled LPD model (1.2), we assume that the jump matrix $T(x, \xi)=\mathrm{I}$ is a $4 \times 4$ unit matrix in Eq.(2.28). In other words, the discrete scattering data $r_{12}=r_{13}=r_{14}=s_{21}=s_{31}=s_{41}=0$, consequently, the unique solution to this special RH problem can be described as

$$
\begin{aligned}
& H_{+}(\xi)=\mathrm{I}+\sum_{m=1}^{N} \sum_{n=1}^{N} \frac{v_{m} \widetilde{v}_{n}\left(P^{-1}\right)_{m n}}{\xi-\widetilde{\xi}_{n}}, \\
& H_{-}(\xi)=\mathrm{I}-\sum_{m=1}^{N} \sum_{n=1}^{N} \frac{v_{m} \widetilde{v}_{n}\left(P^{-1}\right)_{m n}}{\xi-\widetilde{\xi}_{n}},
\end{aligned}
$$

where $P=\left(P_{m n}\right)_{N \times N}$ is a $N \times N$ matrix defined by

$$
P_{m n}=\frac{\widetilde{v}_{m} v_{n}}{\widetilde{\xi}_{m}-\xi_{n}}, 1 \leq m, n \leq N,
$$

therefore, from (3.14a) and (3.14b), we obtain

$$
H_{+}^{(1)}=\sum_{m=1}^{N} \sum_{n=1}^{N} v_{m}\left(P^{-1}\right)_{m n} \widetilde{v}_{n},
$$


choosing $v_{m 0}=\left(a_{m}, b_{m}, c_{m}, d_{m}\right)^{\mathrm{T}}$ and $\rho_{m}=\omega_{m}=0$, from (3.16), we get that the general $\mathrm{N}$-soliton solution for the three-coupled LPD model (1.2) reads

$$
\begin{aligned}
& q_{1}=2 i \sum_{m=1}^{N} \sum_{n=1}^{N} b_{m}^{*} a_{n} e^{\theta_{n}^{*}-\theta_{m}}\left(P^{-1}\right)_{m n}, \\
& q_{2}=2 i \sum_{m=1}^{N} \sum_{n=1}^{N} c_{m}^{*} a_{n} e^{\theta_{n}^{*}-\theta_{m}}\left(P^{-1}\right)_{m n}, \\
& q_{c}=2 i \sum_{m=1}^{N} \sum_{n=1}^{N} d_{m}^{*} a_{n} e^{\theta_{n}^{*}-\theta_{m}}\left(P^{-1}\right)_{m n},
\end{aligned}
$$

where $\theta_{n}=i \xi_{n} x-8 i \varepsilon \xi_{n}^{4} t+2 i \xi_{n}^{2} t, P=\left(P_{m n}\right)_{N \times N}$ is a $N \times N$ matrix with elements given by

$$
P_{m n}=\frac{a_{m}^{*} a_{n} e^{\theta_{m}^{*}+\theta_{n}}+\left(b_{m}^{*} b_{n}+c_{m}^{*} c_{n}+d_{m}^{*} d_{n}\right) e^{-\left(\theta_{m}^{*}+\theta_{n}\right)}}{\xi_{m}^{*}-\xi_{n}}, 1 \leq m, n \leq N
$$

Furthermore, the N-soliton solutions Eq.(3.17a)-(3.17c) can be rewritten as the following determinant ratio form

$$
q_{1}(x, t)=-2 i \frac{\operatorname{det} D_{1}}{\operatorname{det} P}, \quad q_{2}(x, t)=-2 i \frac{\operatorname{det} D_{2}}{\operatorname{det} P}, \quad q_{3}(x, t)=-2 i \frac{\operatorname{det} D_{3}}{\operatorname{det} P},
$$

where the $N \times N$ matrix $P$ is defined by Eq.(3.18), and $D_{1}, D_{2}, D_{3}$ are $(N+1) \times(N+1)$ matrices given by

$$
\begin{aligned}
D_{1} & =\left(\begin{array}{cccc}
P_{11} & \cdots & P_{1 N} & a_{1} e^{-\theta_{1}} \\
\vdots & \vdots & \vdots & \vdots \\
P_{N 1} & \cdots & P_{N N} & a_{N} e^{-\theta_{N}} \\
b_{1}^{*} e^{\theta_{1}^{*}} & \cdots & b_{N}^{*} e^{\theta_{N}^{*}} & 0
\end{array}\right), D_{2}=\left(\begin{array}{cccc}
P_{11} & \cdots & P_{1 N} & a_{1} e^{-\theta_{1}} \\
\vdots & \vdots & \vdots & \vdots \\
P_{N 1} & \cdots & P_{N N} & a_{N} e^{-\theta_{N}} \\
c_{1}^{*} e^{\theta_{1}^{*}} & \cdots & c_{N}^{*} e^{\theta_{N}^{*}} & 0
\end{array}\right) \\
D_{3} & =\left(\begin{array}{cccc}
P_{11} & \cdots & P_{1 N} & a_{1} e^{-\theta_{1}} \\
\vdots & \vdots & \vdots & \vdots \\
P_{N 1} & \cdots & P_{N N} & a_{N} e^{-\theta_{N}} \\
d_{1}^{*} e^{\theta_{1}^{*}} & \cdots & d_{N}^{*} e^{\theta_{N}^{*}} & 0
\end{array}\right)
\end{aligned}
$$




\section{Dynamic behaviors of soliton solutions}

In what follows, we can examine the nonlinear dynamical behaviors of the breather and soliton solutions for the three-coupled LPD model (1.2).

Firstly, as a simple example, choosing $N=1$ in Eqs.(3.17a)-(3.17c) and with Eq.(3.15) gives rise to the following single-soliton solution of the three-coupled LPD model (1.2)

$$
\begin{aligned}
& q_{1}(x, t)=\frac{2 i b_{1}^{*} a_{1} e^{\theta_{1}^{*}-\theta_{1}}\left(\xi_{1}^{*}-\xi_{1}\right)}{\left|a_{1}\right|^{2} e^{\theta_{1}^{*}+\theta_{1}}+\left(\left|b_{1}\right|^{2}+\left|c_{1}\right|^{2}+\left|d_{1}\right|^{2}\right) e^{-\left(\theta_{1}^{*}+\theta_{1}\right)}}, \\
& q_{2}(x, t)=\frac{2 i c_{1}^{*} a_{1} e^{\theta_{1}^{*}-\theta_{1}}\left(\xi_{1}^{*}-\xi_{1}\right)}{\left|a_{1}\right|^{2} e^{\theta_{1}^{*}+\theta_{1}}+\left(\left|b_{1}\right|^{2}+\left|c_{1}\right|^{2}+\left|d_{1}\right|^{2}\right) e^{-\left(\theta_{1}^{*}+\theta_{1}\right)}}, \\
& q_{3}(x, t)=\frac{2 i d_{1}^{*} a_{1} e^{\theta_{1}^{*}-\theta_{1}}\left(\xi_{1}^{*}-\xi_{1}\right)}{\left|a_{1}\right|^{2} e^{\theta_{1}^{*}+\theta_{1}}+\left(\left|b_{1}\right|^{2}+\left|c_{1}\right|^{2}+\left|d_{1}\right|^{2}\right) e^{-\left(\theta_{1}^{*}+\theta_{1}\right)}},
\end{aligned}
$$

where $\theta_{1}=i \xi_{1} x-8 i \varepsilon \xi_{1}^{4} t+2 i \xi_{1}^{2} t$. Let $\xi_{1}=\beta_{1}+i \gamma_{1}, \varepsilon=\frac{1}{2}, a_{1}=1,\left|b_{1}\right|^{2}+\left|c_{1}\right|^{2}+\left|d_{1}\right|^{2}=e^{-2 \eta_{1}}$, the single-soliton solution Eqs.(4.1a)-(4.1c) becomes to

$$
\begin{aligned}
& q_{1}(x, t)=2 b_{1}^{*} \gamma_{1} e^{-2 i\left[\beta_{1} x-4\left(\beta_{1}^{4}-6 \beta_{1}^{2} \gamma_{1}^{2}+\gamma_{1}^{4}\right) t+2\left(\beta_{1}^{2}-\gamma_{1}^{2}\right) t+\eta_{1}\right]} \operatorname{sech} \Delta(x, t), \\
& q_{2}(x, t)=2 c_{1}^{*} \gamma_{1} e^{-2 i\left[\beta_{1} x-4\left(\beta_{1}^{4}-6 \beta_{1}^{2} \gamma_{1}^{2}+\gamma_{1}^{4}\right) t+2\left(\beta_{1}^{2}-\gamma_{1}^{2}\right) t+\eta_{1}\right]} \operatorname{sech} \Delta(x, t), \\
& q_{3}(x, t)=2 d_{1}^{*} \gamma_{1} e^{-2 i\left[\beta_{1} x-4\left(\beta_{1}^{4}-6 \beta_{1}^{2} \gamma_{1}^{2}+\gamma_{1}^{4}\right) t+2\left(\beta_{1}^{2}-\gamma_{1}^{2}\right) t+\eta_{1}\right]} \operatorname{sech} \Delta(x, t),
\end{aligned}
$$

where $\Delta(x, t)=-2 \gamma_{1} x+32 \beta_{1} \gamma_{1}\left(\beta_{1}^{2}-\gamma_{1}^{2}\right) t-8 \beta_{1} \gamma_{1} t+\eta_{1}$.

By choosing different parameters, we can obtain some plots which are displayed to particularly describe the dynamic behaviors for single-soliton solution Eqs.(4.2a)-(4.2c) in the following. Figure 1 illustrates that the single-soliton solution $q_{1}(x, t)$ is a line-soliton, and its widths, amplitudes and velocities remain invariant during the propagation.

Secondly, as a special example, choosing $N=2$ in Eqs.(3.17a)-(3.17c) and with Eq.(3.15) arrives at the following two-soliton solution of the three-coupled LPD model (1.2)

$$
\begin{aligned}
q_{1}(x, t)= & 2 i b_{1}^{*} a_{1} e^{\theta_{1}^{*}-\theta_{1}}\left(P^{-1}\right)_{11}+2 i b_{1}^{*} a_{2} e^{\theta_{1}^{*}-\theta_{2}}\left(P^{-1}\right)_{12} \\
& +2 i b_{2}^{*} a_{1} e^{\theta_{2}^{*}-\theta_{1}}\left(P^{-1}\right)_{21}+2 i b_{2}^{*} a_{2} e^{\theta_{2}^{*}-\theta_{2}}\left(P^{-1}\right)_{22}, \\
q_{2}(x, t)= & 2 i c_{1}^{*} a_{1} e^{\theta_{1}^{*}-\theta_{1}}\left(P^{-1}\right)_{11}+2 i c_{1}^{*} a_{2} e^{\theta_{1}^{*}-\theta_{2}}\left(P^{-1}\right)_{12} \\
& +2 i c_{2}^{*} a_{1} e^{\theta_{2}^{*}-\theta_{1}}\left(P^{-1}\right)_{21}+2 i c_{2}^{*} a_{2} e^{\theta_{2}^{*}-\theta_{2}}\left(P^{-1}\right)_{22}, \\
q_{3}(x, t)= & 2 i d_{1}^{*} a_{1} e^{\theta_{1}^{*}-\theta_{1}}\left(P^{-1}\right)_{11}+2 i d_{1}^{*} a_{2} e^{\theta_{1}^{*}-\theta_{2}}\left(P^{-1}\right)_{12}
\end{aligned}
$$



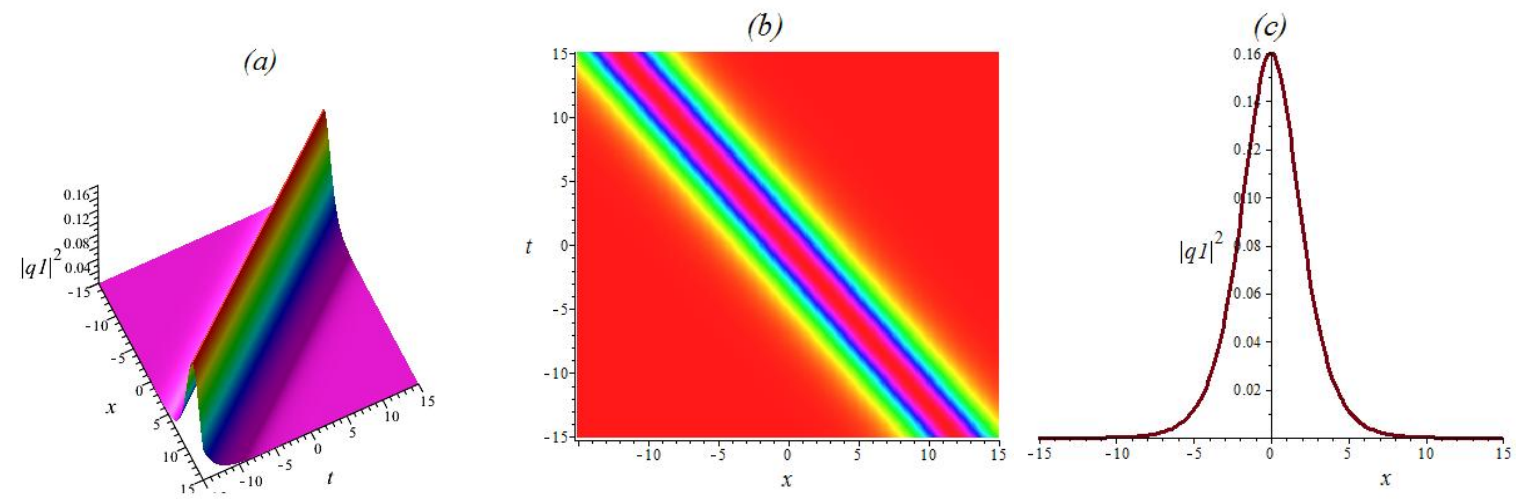

Figure 1: The single-soliton solution (4.2a) $q_{1}(\mathrm{x}, \mathrm{t})$ for three-coupled LPD model (1.2): $\eta_{1}=0, b_{1}=1, \beta_{1}=\frac{1}{5}, \gamma_{1}=-\frac{1}{5}$. (a) Perspective view of modulus of $q_{1}(x, t)$. (b) Density plot of modulus of $q_{1}(x, t)$. (c) The wave propagation along the $x$-axis with $t=0$.

$$
+2 i d_{2}^{*} a_{1} e^{\theta_{2}^{*}-\theta_{1}}\left(P^{-1}\right)_{21}+2 i d_{2}^{*} a_{2} e^{\theta_{2}^{*}-\theta_{2}}\left(P^{-1}\right)_{22},
$$

where $\theta_{l}=i \xi_{l} x-8 i \varepsilon \xi_{l}^{4} t+2 i \xi_{l}^{2} t,(l=1,2)$, and $P=\left(P_{m n}\right)_{2 \times 2}$ is a $2 \times 2$ matrix with the following elements

$$
\begin{aligned}
& P_{11}=\frac{\left|a_{1}\right|^{2} e^{\theta_{1}+\theta_{1}^{*}}+\left(\left|b_{1}\right|^{2}+\left|c_{1}\right|^{2}+\left|d_{1}\right|^{2}\right) e^{-\left(\theta_{1}+\theta_{1}^{*}\right)}}{\xi_{1}^{*}-\xi_{1}}, \\
& P_{12}=\frac{a_{1}^{*} a_{2} e^{\theta_{2}+\theta_{1}^{*}}+\left(b_{1}^{*} b_{2}+c_{1}^{*} c_{2}+d_{1}^{*} d_{2}\right) e^{-\left(\theta_{2}+\theta_{1}^{*}\right)}}{\xi_{1}^{*}-\xi_{2}}, \\
& P_{21}=\frac{a_{2}^{*} a_{1} e^{\theta_{1}+\theta_{2}^{*}}+\left(b_{2}^{*} b_{1}+c_{2}^{*} c_{1}+d_{2}^{*} d_{1}\right) e^{-\left(\theta_{1}+\theta_{2}^{*}\right)}}{\xi_{2}^{*}-\xi_{1}}, \\
& P_{22}=\frac{\left|a_{2}\right|^{2} e^{\theta_{2}+\theta_{2}^{*}}+\left(\left|b_{2}\right|^{2}+\left|c_{2}\right|^{2}+\left|d_{2}\right|^{2}\right) e^{-\left(\theta_{2}+\theta_{2}^{*}\right)}}{\xi_{2}^{*}-\xi_{2}} .
\end{aligned}
$$

Let $\xi_{l}=\beta_{l}+i \gamma_{l},(l=1,2), \varepsilon=\frac{1}{2}, a_{1}=a_{2}=1, b_{1}=b_{2}, c_{1}=c_{2}, d_{1}=d_{2},\left|b_{l}\right|^{2}+\left|c_{l}\right|^{2}+\left|d_{l}\right|^{2}=$ $e^{-2 \eta_{2}}$, the two-soliton solution Eqs.(4.3a)-(4.3c) becomes to

$$
\begin{aligned}
& q_{1}(x, t)=\frac{2 i b_{1}^{*}\left[e^{\theta_{1}^{*}-\theta_{1}} P_{22}-e^{\theta_{2}^{*}-\theta_{1}} P_{21}-e^{\theta_{1}^{*}-\theta_{2}} P_{12}+e^{\theta_{2}^{*}-\theta_{2}} P_{11}\right]}{P_{11} P_{22}-P_{12} P_{21}}, \\
& q_{2}(x, t)=\frac{2 i c_{1}^{*}\left[e^{\theta_{1}^{*}-\theta_{1}} P_{22}-e^{\theta_{2}^{*}-\theta_{1}} P_{21}-e^{\theta_{1}^{*}-\theta_{2}} P_{12}+e^{\theta_{2}^{*}-\theta_{2}} P_{11}\right]}{P_{11} P_{22}-P_{12} P_{21}},
\end{aligned}
$$




$$
q_{3}(x, t)=\frac{2 i d_{1}^{*}\left[e^{\theta_{1}^{*}-\theta_{1}} P_{22}-e^{\theta_{2}^{*}-\theta_{1}} P_{21}-e^{\theta_{1}^{*}-\theta_{2}} P_{12}+e^{\theta_{2}^{*}-\theta_{2}} P_{11}\right]}{P_{11} P_{22}-P_{12} P_{21}},
$$

where

$$
\begin{aligned}
& P_{11}=\frac{i e^{-\eta_{2}}}{\gamma_{1}} \cosh \left(\theta_{1}+\theta_{1}^{*}+\eta_{2}\right), \quad P_{12}=\frac{2 e^{-\eta_{2}}}{\left(\beta_{1}-\beta_{2}\right)-i\left(\gamma_{1}+\gamma_{2}\right)} \cosh \left(\theta_{2}+\theta_{1}^{*}+\eta_{2}\right), \\
& P_{22}=\frac{i e^{-\eta_{2}}}{\gamma_{2}} \cosh \left(\theta_{2}+\theta_{2}^{*}+\eta_{2}\right),
\end{aligned}
$$

By choosing different parameters, we can obtain the interaction solutions Eqs.(4.4a)-(4.4c) which are displayed in Figure 2 and Figure 3. Figure 2 illustrates that soliton transmission, and the two solitons moving over each other, and their polarizations remaining unchanged. Figure 3 illustrates that soliton reflection produces the breather soliton solution.

(a)

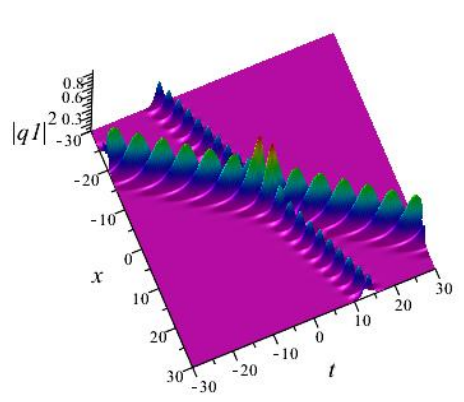

(b)

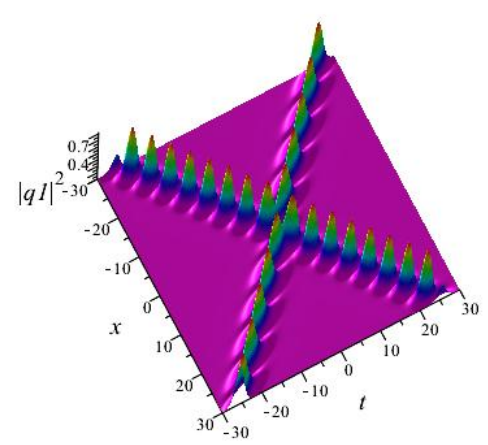

Figure 2: The two-soliton solution (4.4a) $q_{1}(x, t)$ for three-coupled LPD model (1.2) by choosing suitable parameters: (a) $\eta_{2}=0, b_{1}=1, \beta_{1}=-0.3, \beta_{2}=0.4, \gamma_{1}=-0.3, \gamma_{2}=0.3$. (b) $\eta_{2}=0, b_{1}=1, \beta_{1}=0.7, \beta_{2}=-0.5, \gamma_{1}=0.2, \gamma_{2}=-0.3$.

Finally, as another special example, choosing $N=3$ in Eqs.(3.17a)-(3.17c) and with Eq.(3.15) arrives at the following three-soliton solution of the three-coupled LPD model (1.2)

$$
\begin{aligned}
q_{1}(x, t) & =2 i b_{1}^{*} a_{1} e^{\theta_{1}^{*}-\theta_{1}}\left(P^{-1}\right)_{11}+2 i b_{1}^{*} a_{2} e^{\theta_{1}^{*}-\theta_{2}}\left(P^{-1}\right)_{12}+2 i b_{1}^{*} a_{3} e^{\theta_{1}^{*}-\theta_{3}}\left(P^{-1}\right)_{13} \\
& +2 i b_{2}^{*} a_{1} e^{\theta_{2}^{*}-\theta_{1}}\left(P^{-1}\right)_{21}+2 i b_{2}^{*} a_{2} e^{\theta_{2}^{*}-\theta_{2}}\left(P^{-1}\right)_{22}+2 i b_{2}^{*} a_{3} e^{\theta_{2}^{*}-\theta_{3}}\left(P^{-1}\right)_{23}
\end{aligned}
$$



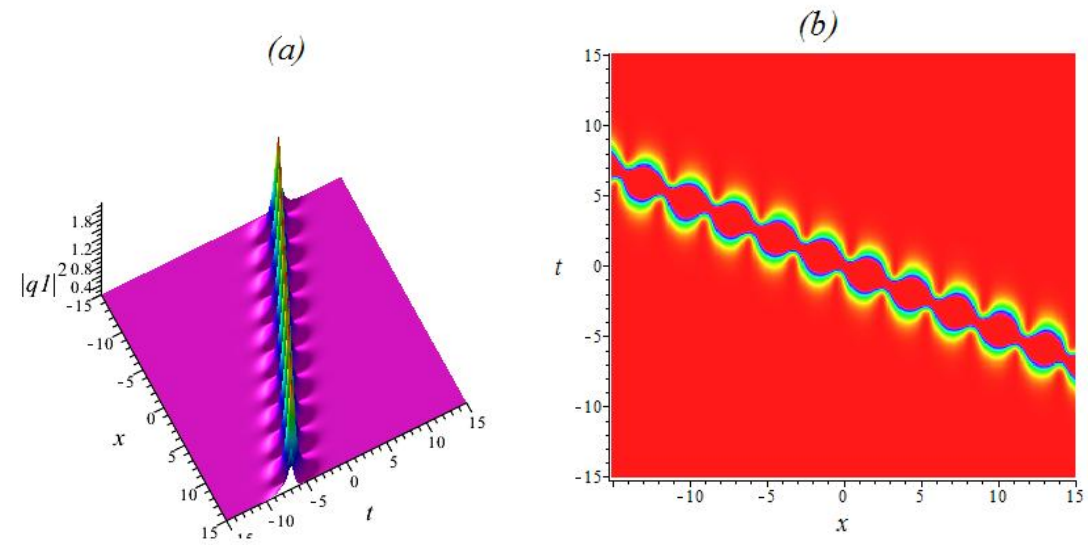

Figure 3: The breather soliton solution (4.4a) $q_{1}(x, t)$ for three-coupled LPD model (1.2) by choosing suitable parameters: $\eta_{2}=0, b_{1}=0.5-0.5 i, \beta_{1}=0.4, \beta_{2}=-0.7, \gamma_{1}=0.5, \gamma_{2}=$ -0.2 . (a) Perspective view of modulus of breather soliton $q_{1}(x, t)$. (b) Density plot of modulus of breather soliton $q_{1}(x, t)$.

$$
\begin{aligned}
& +2 i b_{3}^{*} a_{1} e^{\theta_{3}^{*}-\theta_{1}}\left(P^{-1}\right)_{31}+2 i b_{3}^{*} a_{2} e^{\theta_{3}^{*}-\theta_{2}}\left(P^{-1}\right)_{32}+2 i b_{3}^{*} a_{3} e^{\theta_{3}^{*}-\theta_{3}}\left(P^{-1}\right)_{33}, \\
q_{2}(x, t) & =2 i c_{1}^{*} a_{1} e^{\theta_{1}^{*}-\theta_{1}}\left(P^{-1}\right)_{11}+2 i c_{1}^{*} a_{2} e^{\theta_{1}^{*}-\theta_{2}}\left(P^{-1}\right)_{12}+2 i c_{1}^{*} a_{3} e^{\theta_{1}^{*}-\theta_{3}}\left(P^{-1}\right)_{13} \\
& +2 i c_{2}^{*} a_{1} e^{\theta_{2}^{*}-\theta_{1}}\left(P^{-1}\right)_{21}+2 i c_{2}^{*} a_{2} e^{\theta_{2}^{*}-\theta_{2}}\left(P^{-1}\right)_{22}+2 i c_{2}^{*} a_{3} e^{\theta_{2}^{*}-\theta_{3}}\left(P^{-1}\right)_{23} \\
& +2 i c_{3}^{*} a_{1} e^{\theta_{3}^{*}-\theta_{1}}\left(P^{-1}\right)_{31}+2 i c_{3}^{*} a_{2} e^{\theta_{3}^{*}-\theta_{2}}\left(P^{-1}\right)_{32}+2 i c_{3}^{*} a_{3} e^{\theta_{3}^{*}-\theta_{3}}\left(P^{-1}\right)_{33}, \\
q_{3}(x, t) & =2 i d_{1}^{*} a_{1} e^{\theta_{1}^{*}-\theta_{1}}\left(P^{-1}\right)_{11}+2 i d_{1}^{*} a_{2} e^{\theta_{1}^{*}-\theta_{2}}\left(P^{-1}\right)_{12}+2 i d_{1}^{*} a_{3} e^{\theta_{1}^{*}-\theta_{3}}\left(P^{-1}\right)_{13} \\
& +2 i d_{2}^{*} a_{1} e^{\theta_{2}^{*}-\theta_{1}}\left(P^{-1}\right)_{21}+2 i d_{2}^{*} a_{2} e^{\theta_{2}^{*}-\theta_{2}}\left(P^{-1}\right)_{22}+2 i d_{2}^{*} a_{3} e^{\theta_{2}^{*}-\theta_{3}}\left(P^{-1}\right)_{23} \\
& +2 i d_{3}^{*} a_{1} e^{\theta_{3}^{*}-\theta_{1}}\left(P^{-1}\right)_{31}+2 i d_{3}^{*} a_{2} e^{\theta_{3}^{*}-\theta_{2}}\left(P^{-1}\right)_{32}+2 i d_{3}^{*} a_{3} e^{\theta_{3}^{*}-\theta_{3}}\left(P^{-1}\right)_{33},
\end{aligned}
$$

where $\theta_{j}=i \xi_{j} x-8 i \varepsilon \xi_{j}^{4} t+2 i \xi_{j}^{2} t,(j=1,2,3)$, and $M=\left(P_{m n}\right)_{3 \times 3}$ is a $3 \times 3$ matrix with following elements

$$
\begin{aligned}
& P_{11}=\frac{\left|a_{1}\right|^{2} e^{\theta_{1}+\theta_{1}^{*}}+\left(\left|b_{1}\right|^{2}+\left|c_{1}\right|^{2}+\left|d_{1}\right|^{2}\right) e^{-\left(\theta_{1}+\theta_{1}^{*}\right)}}{\xi_{1}^{*}-\xi_{1}}, \\
& P_{12}=\frac{a_{1}^{*} a_{2} e^{\theta_{2}+\theta_{1}^{*}}+\left(b_{1}^{*} b_{2}+c_{1}^{*} c_{2}+d_{1}^{*} d_{2}\right) e^{-\left(\theta_{2}+\theta_{1}^{*}\right)}}{\xi_{1}^{*}-\xi_{2}}, \\
& P_{13}=\frac{a_{1}^{*} a_{3} e^{\theta_{2}+\theta_{1}^{*}}+\left(b_{1}^{*} b_{3}+c_{1}^{*} c_{3}+d_{1}^{*} d_{3}\right) e^{-\left(\theta_{3}+\theta_{1}^{*}\right)}}{\xi_{1}^{*}-\xi_{3}},
\end{aligned}
$$




$$
\begin{aligned}
& P_{21}=\frac{a_{2}^{*} a_{1} e^{\theta_{1}+\theta_{2}^{*}}+\left(b_{2}^{*} b_{1}+c_{2}^{*} c_{1}+d_{2}^{*} d_{1}\right) e^{-\left(\theta_{1}+\theta_{2}^{*}\right)}}{\xi_{2}^{*}-\xi_{1}}, \\
& P_{22}=\frac{\left|a_{2}\right|^{2} e^{\theta_{2}+\theta_{2}^{*}}+\left(\left|b_{2}\right|^{2}+\left|c_{2}\right|^{2}+\left|d_{2}\right|^{2}\right) e^{-\left(\theta_{2}+\theta_{2}^{*}\right)}}{\xi_{2}^{*}-\xi_{2}}, \\
& P_{23}=\frac{a_{2}^{*} a_{3} e^{\theta_{3}+\theta_{2}^{*}}+\left(b_{2}^{*} b_{3}+c_{2}^{*} c_{3}+d_{2}^{*} d_{3}\right) e^{-\left(\theta_{3}+\theta_{2}^{*}\right)}}{\xi_{2}^{*}-\xi_{3}}, \\
& P_{31}=\frac{a_{3}^{*} a_{1} e^{\theta_{1}+\theta_{3}^{*}}+\left(b_{3}^{*} b_{1}+c_{3}^{*} c_{1}+d_{3}^{*} d_{1}\right) e^{-\left(\theta_{1}+\theta_{3}^{*}\right)}}{\xi_{3}^{*}-\xi_{1}}, \\
& P_{32}=\frac{a_{3}^{*} a_{2} e^{\theta_{2}+\theta_{3}^{*}}+\left(b_{3}^{*} b_{2}+c_{3}^{*} c_{2}+d_{3}^{*} d_{2}\right) e^{-\left(\theta_{2}+\theta_{3}^{*}\right)}}{\xi_{3}^{*}-\xi_{2}}, \\
& P_{33}=\frac{\left|a_{3}\right|^{2} e^{\theta_{3}+\theta_{3}^{*}}+\left(\left|b_{3}\right|^{2}+\left|c_{3}\right|^{2}+\left|d_{3}\right|^{2}\right) e^{-\left(\theta_{3}+\theta_{3}^{*}\right)}}{\xi_{3}^{*}-\xi_{3}} .
\end{aligned}
$$

Let $\xi_{j}=\beta_{j}+i \gamma_{j},(j=1,2,3), \varepsilon=\frac{1}{2}, a_{1}=a_{2}=a_{3}=1, b_{1}=b_{2}=b_{3}, c_{1}=c_{2}=c_{3}$, $d_{1}=d_{2}=d_{3},\left|b_{j}\right|^{2}+\left|c_{j}\right|^{2}+\left|d_{j}\right|^{2}=e^{-2 \eta_{3}}$, by choosing different parameters, we can obtain that the interaction solutions Eqs.(4.5a)-(4.5c) are displayed in Figure 4 and Figure 5. Figure 4 illustrates that soliton transmission, and the three solitons interact each other. Figure 5 illustrates that soliton reflection produces the breather and soliton solution.

\section{$5 \quad$ Discussions and conclusions}

Indeed, as a promotion, the integrable three-coupled LPD model (1.2) can be extended to the integrable generalized N-component LPD model as follows:

$$
\begin{aligned}
i q_{\alpha, t} & +q_{\alpha, x x}+2 \sum_{k=1}^{N}\left|q_{k}\right|^{2} q_{\alpha}+\varepsilon\left[q_{\alpha, x x x x}+2 \sum_{k=1}^{N}\left|q_{k, x}\right|^{2} q_{\alpha}\right. \\
& +2 \sum_{k=1}^{N} q_{k} q_{k, x}^{*} q_{\alpha, x}+6 \sum_{k=1}^{N} q_{k}^{*} q_{k, x} q_{\alpha, x}+4 \sum_{k=1}^{N}\left|q_{k}\right|^{2} q_{\alpha, x x} \\
& \left.+4 \sum_{k=1}^{N} q_{k}^{*} q_{k, x x} q_{\alpha}+2 \sum_{k=1}^{N} q_{k} q_{k, x x}^{*} q_{\alpha}+6\left(\sum_{k=1}^{N}\left|q_{k}\right|^{2}\right)^{2} q_{\alpha}\right]=0,
\end{aligned}
$$

where $q_{\alpha}(x, t),(\alpha=1,2, \ldots, N)$ represents amplitude of molecular excitation in the $\alpha$ th spine, $\varepsilon$ is a real parameter which stands for the strength of higher-order linear and 

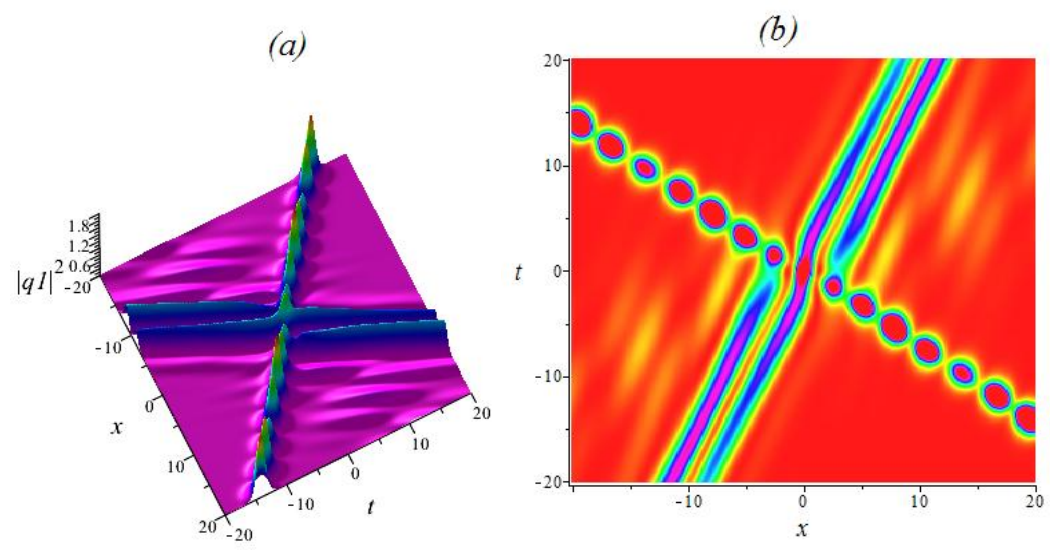

Figure 4: The three-soliton solution (4.5a) $q_{1}(x, t)$ for three-coupled LPD model (1.2) by choosing suitable parameters: $\eta_{3}=0, b_{1}=b_{2}=b_{3}=1, \beta_{1}=0.65, \beta_{2}=-0.5, \beta_{3}=0.3, \gamma_{1}=$ $-0.05, \gamma_{2}=0.25, \gamma_{3}=0.35$. (a) Perspective view of modulus of three-soliton $q_{1}(x, t)$. (b) Density plot of modulus of breather soliton $q_{1}(x, t)$.
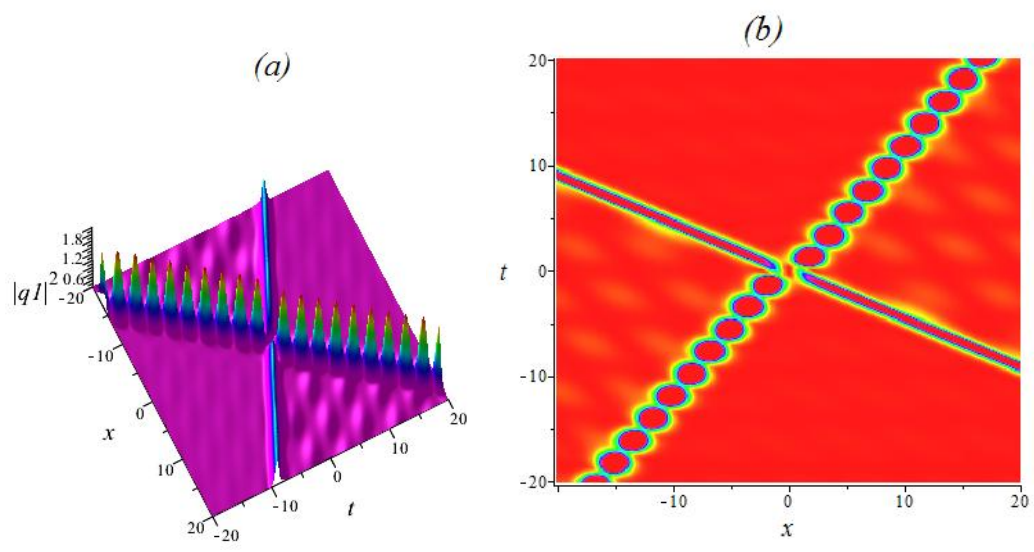

Figure 5: The breather soliton solution (4.5a) $q_{1}(x, t)$ for three-coupled LPD model (1.2) by choosing suitable parameters: $\eta_{3}=0, b_{1}=b_{2}=b_{3}=1, \beta_{1}=0.4, \beta_{2}=-0.1, \beta_{3}=0.1, \gamma_{1}=$ $-0.5, \gamma_{2}=0.5, \gamma_{3}=0.01$. (a) Perspective view of modulus of three-soliton $q_{1}(x, t)$. (b) Density plot of modulus of breather soliton $q_{1}(x, t)$. 
nonlinear effects. Let $\mathbf{q}=\left(q_{1}, q_{2}, \ldots, q_{N}\right)^{T}$, Eq.(5.1) has the following vector form

$$
\begin{aligned}
i \mathbf{q}_{t}+ & \mathbf{q}_{x x}+2 \mathbf{q} \mathbf{q}^{\dagger} \mathbf{q}+\varepsilon\left[\mathbf{q}_{x x x x}+2 \mathbf{q}_{x} \mathbf{q}_{x}^{\dagger} \mathbf{q}+2 \mathbf{q} \mathbf{q}_{x}^{\dagger} \mathbf{q}_{x}\right. \\
& \left.+6 \mathbf{q}_{x} \mathbf{q}^{\dagger} \mathbf{q}_{x}+4 \mathbf{q} \mathbf{q}^{\dagger} \mathbf{q}_{x x}+4 \mathbf{q}_{x x} \mathbf{q}^{\dagger} \mathbf{q}+2 \mathbf{q} \mathbf{q}_{x x}^{\dagger} \mathbf{q}+6\left(\mathbf{q} \mathbf{q}^{\dagger}\right)^{2} \mathbf{q}\right]=0
\end{aligned}
$$

Eq.(5.1) possesses the following $(N+1) \times(N+1)$ matrix spectral problem

$$
\begin{aligned}
& \Phi_{x}=[i \xi \Lambda+\widetilde{M}(x, t)] \Phi, \\
& \Phi_{t}=\left[-8 i \varepsilon \xi^{4} \Lambda+2 i \xi^{2} \Lambda+\widetilde{Q}(x, t, \xi)\right] \Phi,
\end{aligned}
$$

where $\xi$ is a complex spectral parameter, $\Lambda, \widetilde{M}(x, t)$ are $(N+1) \times(N+1)$ matrices defined by

$$
\Lambda=\left(\begin{array}{cc}
1 & \mathbf{0}_{1 \times N} \\
\mathbf{0}_{1 \times N} & -\mathbf{I}_{1 \times N}
\end{array}\right), \quad \widetilde{M}(x, t)=\left(\begin{array}{cc}
0 & \mathbf{q}^{\dagger} \\
\mathbf{q} & \mathbf{0}_{N \times N}
\end{array}\right)
$$

and $\widetilde{Q}(x, t, \xi)$ is a $(N+1) \times(N+1)$ matrix as follows

$$
\begin{aligned}
\widetilde{Q}(x, t, \xi)= & -8 i \varepsilon \widetilde{M} \xi^{3}+4 \varepsilon\left(i \widetilde{M}^{2} \Lambda-\Lambda \widetilde{M}_{x}\right) \xi^{2} \\
& +2\left(i \varepsilon \widetilde{M}_{x x}+2 i \varepsilon \widetilde{M}^{3}-\varepsilon\left(\widetilde{M} \widetilde{M}_{x}-\widetilde{M}_{x} \widetilde{M}\right)+i \widetilde{M}\right) \xi \\
& +\varepsilon \Lambda \widetilde{M}_{x x x}-i \varepsilon \Lambda\left(\widetilde{M} \widetilde{M}_{x x}+\widetilde{M}_{x x} \widetilde{M}\right)-3 i \varepsilon \Lambda \widetilde{M}^{4}-i \Lambda \widetilde{M}^{2} \\
& +i \varepsilon \widetilde{M}_{x}^{2} \Lambda+3 \varepsilon \Lambda\left(\widetilde{M}^{2} \widetilde{M}_{x}+\widetilde{M}_{x} \widetilde{M}^{2}\right)+\Lambda \widetilde{M}_{x}
\end{aligned}
$$

Accordingly, we can also examine the N-soliton solutions to the integrable generalized Ncomponent LPD model (5.1) by the same way in Section 3. However, we don't report them here since the procedure is mechanical.

In this work, we utilize the RH approach to study the three-coupled LPD model (1.2). By constructing a special matrix $\mathrm{RH}$ problem, we have obtained the multi-soliton solution of the LPD model (1.2). In addition, some graphical analysis gives the dynamic characteristics of the soliton solution, including the interaction of single soliton, two-solitons, three-soliton and breather soliton solutions. It is hoped that our results can help enrich and explain other nonlinear integrable models. 
Acknowledgement The authors would like to express our sincere thanks to every member in our discussion group for their valuable comments.

Funding This study was funded by National Natural Science Foundation of China (grant numbers 11675146, 11835011 and 11975145), by Natural Science Foundation of Anhui Province (grant number 2108085QA09), by Postdoctoral Fund of Zhejiang Normal University (grant number ZC304021909).

Data Availability Statement The authors confirm that the data supporting the findings of this study are available within the article.

Conflict of interest The authors declare that they have no conflict of interest.

\section{References}

[1] Gardner, C.S., Green, J.M., Kruskal, M.D., Miüra, R.M.: Method for Solving the KortewegdeVries Equation, Phys. Rev. Lett. 19, 1095-1097 (1967)

[2] Hirota, R.: Exact solution of the Korteweg-de Vries equation for multiple collisions of solitons, Phys. Rev. Lett. 27, 1192-1194 (1971)

[3] Wahlquist, H.D., Estabrook, F.B.: Bäcklund transformation for solutions of the Korteweg-de Vries equation, Phys. Rev. Lett. 31, 1386-1390 (1973)

[4] Matveev, V.B., Salle, M.A.: Darboux Transformations and Solitons. Springer, Berlin, (1991)

[5] Lou, S.Y.: A note on the new similarity reductions of the Boussinesq equation, Phys. Lett. A 151, 133-135 (1990)

[6] Li, J.B., Liu, Z.R.: Smooth and non-smooth traveling waves in a nonlinearly dispersive equation, Appl. Math. Model. 25, 41-56 (2000)

[7] Fan, E.G.: Uniformly constructing a series of explicit exact solutions to nonlinear equations in mathematical physics, Chaos Soliton Fract. 16, 819-839 (2003)

[8] Jin, X.W., Lin, J.: Rogue wave, interaction solutions to the KMM system, J. Magn. Magn. Mater. 502, 166590 (2020). 
[9] Guo, B.L., Ling, L.M., Riemann-Hilbert approach and N-soliton formula for coupled derivative Schrödinger equation, J. Math. Phys. 53, 073506 (2012)

[10] Wang, D.S., Yin, S.J., Liu, Y.F.: Integrability and bright soliton solutions to the coupled nonlinear Schrödinger equation with higher-order effects, Appl. Math. Commun. 229, 296309 (2014)

[11] Wang, Z., Qiao, Z.J.: Riemann-Hilbert approach for the FQXL model: A generalized Camassa-Holm equation with cubic and quadratic nonlinearity, J. Math. Phys. 57, 073505 (2016)

[12] Zhang, Y.S., Cheng, Y., He, J.S.: Riemann-Hilbert method and N-soliton for two-component Gerdjikov-Ivanov equation, J. Nonlinear Math. Phy. 24, 210-223 (2017)

[13] Hu, J., Xu, J., Yu, G.F.: Riemann-Hilbert approach and N-soliton formula for a higher-order Chen-Lee-Liu equation, J. Nonlinear Math. Phy. 25, 633-649 (2018)

[14] Ma, W.X.: Riemann-Hilbert problems and N-soliton solutions for a coupled mKdV system, J. Geom. Phys. 132, 45-54 (2018)

[15] Ma, W.X.: Application of the Riemann-Hilbert approach to the multicomponent AKNS integrable hierarchies, Nonlinear Anal. Real. 47, 1-17 (2019)

[16] Ma, W.X.: The inverse scattering transform and soliton solutions of a combined modified Korteweg-de Vries equation, J. Math. Anal. Appl. 471, 796-811 (2019)

[17] Ma, W.X.: Riemann-Hilbert problems and soliton solutions of a multicomponent mKdV system and its reduction, Math. Method. Appl. Sci. 42, 1099-1113 (2019)

[18] Zhang, W.G., Yao, Q., Bo, G.Q.: Two-soliton solutions of the complex short pulse equation via Riemann-Hilbert approach, Appl. Math. Lett. 98, 263-270 (2019)

[19] Ma, X., Xia, T.C., Riemann-Hilbert Approach and N-soliton solutions for the generalized nonlinear Schrödinger equation, Phys. Scr. 94(9), 095203 (2019)

[20] Xu, S.Q., Li, R.M., Geng, X.G.: Riemann-Hilbert Method for the Three-Component SasaSatsuma Equation and Its N-Soliton Solutions, Rep. Math. Phys. 85(1), 77-103 (2020)

[21] Yang, J.J., Tian, S.F., Peng, W.Q., Zhang, T.T.: The N-coupled higher-order nonlinear Schrödinger equation: Riemann-Hilbert problem and multi-soliton solutions. Math. Meth. Appl. Sci. 43(5), 2458-2472 (2020)

[22] Wang, J., Su, T., Geng, X.G., Li, R.M.: Riemann-Hilbert approach and N-soliton solutions for a new two-component Sasa-Satsuma equation, Nonlinear Dyn. 101(1), 597-609 (2020) 
[23] Liu, N., Guo, B.L., Solitons and rogue waves of the quartic nonlinear Schrödinger equation by Riemann-Hilbert approach, Nonlinear Dyn. 100(1), 629-646 (2020)

[24] Zhang, J.B., Zhang, Z.X.: A Riemann-Hilbert Approach to the Multicomponent KaupNewell Equation, Adv. Math. Phys. 2020, 1-7 (2020)

[25] Guo, H.D,. Xia, T.C.: Multi-soliton solutions for a higher-order coupled nonlinear Schrödinger system in an optical fiber via Riemann-Hilbert approach, Nonlinear Dyn. 103(2), 1805-1816 (2021)

[26] Li, J., Xia, T.C.: A Riemann-Hilbert approach to the Kundu-nonlinear Schrödinger equation and its multi-component generalization, J. Math. Anal. Appl. 500, 125109 (2021)

[27] Li, Y., Li, J., Wang, R.Q.: Multi-soliton solutions of the N-component nonlinear Schrödinger equations via Riemann-Hilbert approach, Nonlinear Dyn. 105, 1765-1772 (2021)

[28] Hu, B.B., Xia, T.C., Zhang, N., Wang, J.B.: Initial-boundary value problems for the coupled higher-order nonlinear Schrödinger equations on the half-line, Int. J. Nonlin. Sci. Num. 19, 83-92 (2018)

[29] Yan, Z.Y.: Initial-boundary value problem for an integrable spin-1 Gross-Pitaevskii system with a $4 \times 4$ Lax pair on a finite interval, J. Math. Phys. 60, 1-70 (2019)

[30] Hu, B.B., Zhang, L., Xia, T.C., Zhang, N.: On the Riemann-Hilbert problem of the Kundu equation. Appl. Math. Comput. 381, 125262 (2020)

[31] Hu, B.B., Zhang, L., Xia, T.C.: On the Riemann-Hilbert problem of a generalized derivative nonlinear Schrödinger equation. Commun. Theor. Phys. 73, 015002 (2021)

[32] Hu, B.B., Zhang, L., Zhang, N.: On the Riemann-Hilbert problem for the mixed ChenLee-Liu derivative nonlinear Schrödinger equation. J. Comput. Appl. Math. 390, 113393 (2021)

[33] Deift, P., Zhou, X.: A steepest descent method for oscillatory Riemann-Hilbert problems, Ann. Math. 137, 295-368 (1993)

[34] Wang, D.S., Guo, B.L., Wang, X.L.: Long-time asymptotics of the focusing Kundu-Eckhaus equation with nonzero boundary conditions, J. Differ. Equations, 266, 5209-5253 (2019)

[35] Lakshmanan, M., Porsezian, K., Daniel, M.: Effect of discreteness on the continuum limit of the Heisenberg spin chain, Phys. Lett. A 133, 483-488 (1988) 
[36] Porsezian, K., Daniel, M., Lakshmanan, M.: On the integrability aspects of the onedimensional classical continuum isotropic biquadratic Heisenberg spin chain, J. Math. Phys. 33, 1807-1816 (1992)

[37] Saravana Veni, S., Latha, M.M.: A generalized Davydov model with interspine coupling and its integrable discretization, Phys. Scr. 86, 025003 (2012)

[38] Sun, W.R., Tian, B., Wang, Y.F., Zhen, H.L.: Soliton excitations and interactions for the three-coupled fourth-order nonlinear Schrödinger equations in the alpha helical proteins, Eur. Phys. J. D 69, 146 (2015)

[39] Du, Z., Tian, B., Chai, H.P., Zhao, X.H.: Lax pair, Darboux transformation and rogue waves for the three-coupled fourth-order nonlinear Schrödinger system in an alpha helical protein, Wave. Random. Complex. (2019) DOI:10.1080/17455030.2019.1644466.

[40] Peng, W.Q., Tian, S.F., Wang, X.B., Zhang, T.T., Fang, Y.: Riemann-Hilbert method and multi-soliton solutions for three-component coupled nonlinear Schrödinger equations, J. Geom. Phys. 146, 103508 (2019)

[41] Yan, Z.Y.: An initial-boundary value problem of the general three-component nonlinear Schrödinger equation with a $4 \times 4$ lax pair on a finite interval, arXiv:1704.08561, (2017) 